\title{
Neuroprotective therapies for multiple sclerosis and other demyelinating diseases

Pablo Villoslada ${ }^{1,2}$

\begin{abstract}
Damage to the Central Nervous Systems (CNS) in Multiple Sclerosis (MS) seems to be mainly due to chronic inflammation of the CNS with superimposed bouts of inflammatory activity by the adaptive immune system. The immune mediated damage can be amplified by neurodegenerative mechanisms in damaged axons including anterograde or retrograde axonal or transynaptic degeneration, synaptic pruning and neuronal or oligodendrocyte death. As such, it is highly unlikely that CNS damage can be prevented using only immunomodulatory drugs. For this reason, neuroprotection, aimed at preventing axonal, neuronal, myelin, and oligodendrocyte damage and cell death in the presence of this toxic microenvironment is highly pursued in MS and other demyelinating diseases. Neuroprotective strategies target different processes including oxidative stress, ionic imbalance (sodium, potassium or calcium), energy depletion, trophic factor support, metabolites balance, excitotoxicity, apoptosis, remyelination, etc. Although none of these strategies have translated into approved drugs to date, improvement in the understanding of underlying biology, in the design of clinical trials specific for assessing neuroprotection, and new technologies for developing novel therapies for neuroprotection suggest a new avenue for treating MS, Optic Neuritis or Neuromyelitis Optica (NMO). Several of these therapies are now entering clinical phases and if successful, such strategies would improve patients' quality of life, and will be even more critical for patients with progressive MS. In the event that such therapies target natural repair mechanisms rather than disease specific processes, they can potentially be useful for other brain diseases such as stroke, neurodegenerative diseases, brain trauma or epilepsy.
\end{abstract}

Keywords: Multiple sclerosis, Neuromyelitis optica, Demyelinating diseases, Neuroprotection, Trophic factors, Axonal damage, Remyelination

\section{Background}

The central nervous system is highly sensitive to damage: the role of neuroprotection

The Central Nervous System (CNS) is especially sensitive to damage compared to other tissues because of its highly specialized structure and function; it is composed of billions of neurons making both long and short-range connections, requires high energy and metabolite consumption, and has significant post-damage repair restrictions. Brain connections are made in a highly complex and synchronized process during development and are refined with training [1]. Once defined, brain connectivity is fixed by myelination and other processes in order to preserve memory and function $[1,2]$. For this reason,

\footnotetext{
Correspondence: pvilloslada@clinic.ub.es

${ }^{1}$ Center of Neuroimmunology, Institut d'Investigacions Biomèdiques August Pi i Sunyer (IDIBAPS), Centre Cellex 3A, Casanova 145, Barcelona 08036, Spain ${ }^{2}$ Department of Neurology, University of California, San Francisco, USA
}

there are significant limitations for promoting neuronal network regeneration in adults after damage (e.g. presence of axonal growth inhibitory molecules such as neurite outgrowth inhibitor A (Nogo-A)). Nevertheless, even if regenerative therapy for the CNS is highly sought after, an intermediate, longer-term promising alternative approach is neuroprotection [3].

After insults such as ischemia, inflammation or excitotoxicity, neurons and axons may suffer significant damage, resulting in oxidative damage of DNA and proteins, reduced energy production, imbalance of ionic homeostasis and ion channel functioning, endoplasmic reticulum impairment and protein folding degradation or microtubule mediated axonal transport impairment. Due to the high level energetic and functional requirements that neurons have for maintaining long-distance nerve conduction (with axons up to $0.5 \mathrm{~m}$ long in the corticospinal 
tract), neuronal malfunction can trigger self-destruction processes such as apoptosis, autophagia, synaptic pruning and many other forms of neuronal cell death [4-6]. Furthermore, damaged axons can trigger an active process of axonal degeneration regulated by levels of nicotinamide adenine dinucleotide (NAD). Axonal degeneration is a process different from apoptosis, which results in acute axonal transection and chronic anterograde (Wallerian) or retrograde degeneration. This process is regulated by several key molecules such as nicotinamide nucleotide adenylyltransferase 2 (NMNAT2), Sterile Alpha And TIR Motif Containing 1 (Sarm1) and phosphate starvation response 1 (PHR1) which regulates levels of NAD, or downstream steps regulated by c-Jun $\mathrm{N}$-terminal kinases (JNK), glycogen synthase kinase 3 (GSK3) or inhibitor of kappa B kinase (IKK) converging in mitochondria and energy dysfunction and calpains activation leading to calcium imbalance [7, 8]. Additionally, myelin is highly susceptible to damage in the white matter because oligodendrocytes are also high-energy demanding cells (myelin turn-over is around one month) but the blood supply prioritizes grey over white matter [6]. Moreover, lipid structures such as myelin are highly susceptible to damage by immune mediators and oxidative stress [9]. In this context, inflammatory, ischemic or degenerative insults can induce either direct damage of the neurons (e.g. necrosis) or delayed degenerative processes that are triggered once surviving neurons identify they can no longer maintain their function and initiate different forms of cell death, axonal degeneration or synaptic pruning [10]. Another feature to take into account when managing neurological damage is the plastic and redundant nature of the CNS, which explain why damage of many CNS regions are not eloquent at the clinical level. This implies that CNS damage need to surpasse a given threshold of damage in order to translate to clinical symptoms, which prevents close monitoring of CNS damage by clinical assessment and cause delayed diagnosis or disability monitoring. All these facts, including sensitivity of neural networks to damage, poor regenerative ability, and late diagnosis of CNS damage, support neuroprotection as an important therapeutic strategy for decreasing the burden of neurological diseases.

\section{Neuroprotective strategies}

Almost every mechanism of damage identified in brain diseases has been proposed as a therapeutic target for neuroprotection (Fig. 1). Several biological processes specific to the CNS (e.g. trophic factor signaling, axonal guidance, myelin formation) or critical for neurons (e.g. apoptosis, energetic supply, ionic balance) have been mimicked with experimental therapies $[3,11]$. In addition, several regenerative therapies, such as stem cells, may provide some benefits via neuroprotective effects, including the release of trophic factors, suppressing local inflammation or promoting a microenvironment supporting the survival of neurons, axons and oligodendrocytes [12]. Finally, secondary neuroprotection can be achieved by the reduction of the insult such as restoring blood supply in ischemia, decreasing excitotoxicity by reducing epileptogenic activity in seizures or decreasing CNS inflammation with the use of immunomodulatory drugs (e.g. glatiramer acetate, fingolimod, dimethyl-fumarate or laquinimod) in the case of MS [13-16].

Table 1 displays a list of several therapeutic strategies being pursued for neuroprotection. In the pursuit for neuroprotective strategies, trophic factors are proposed as the Holy Grail $[17,18]$. Rather than coding for all neuronal connections during development, evolution developed the trophic factor strategy, which regulates neuronal survival and connection maintenance with the release of trophic factors from the target cell to the projecting neuron. For this reason, trophic factors activate a set of signaling pathways in neurons, such as Phosphoinositide 3-kinase (PI3K), Mitogen-activated protein kinase kinase (MAPKK), Nuclear factor- $k B$ (NFKB) and others that stop apoptosis, promote cell survival and differentiation and trigger other beneficial effects such as decreasing oxidative stress, regulating ion channels, etc. [19]. Several growth factors have been tested in animal models or clinical trials including neurotrophins (nerve growth factor, brain-derived nerve factor, neurotrophin-3), insulin-growth factor (IGF-1), neurocytokines (cilliary-neurotrophic factor, leukemia inhibitor factor, interleukin-6), and glial-derived nerve factor family (erythropoietin, etc.) [20, 21]. They were tested in trials of peripheral neuropathy (diabetes or adquired immunodeficiency syndrome) or neurodegenerative diseases (Alzheimer disease, Parkinson disease, Huntington disease, Amyotrophic Lateral Sclerosis (ALS)) using human recombinant proteins delivered intravenously, intrathecaly or using engineered cells or gene therapy vectors [22-25]. Lack of success to date for the use of trophic factors to prevent CNS damage does not preclude the usefulness of trophic factors as a therapeutic target. Lack of efficacy was attributed to poor pharmacological properties of the recombinant proteins to enter the CNS and reach the target neurons, inadequate clinical trial design, such as testing patients in late stages when neuronal death is massive and using insensitive clinical or imaging outcomes, or side effects that limited dosing and patient exposition [26, 27]. However, the trophic factor pathways are at the core of cellular processes promoting neuronal and oligodendrocyte survival and for this reason are worth pursuing to see if activation of these pathways using different strategies might prevent permanent CNS damage.

Energy depletion and mitochondria dysfunction are another key factor in promoting neuronal degeneration 


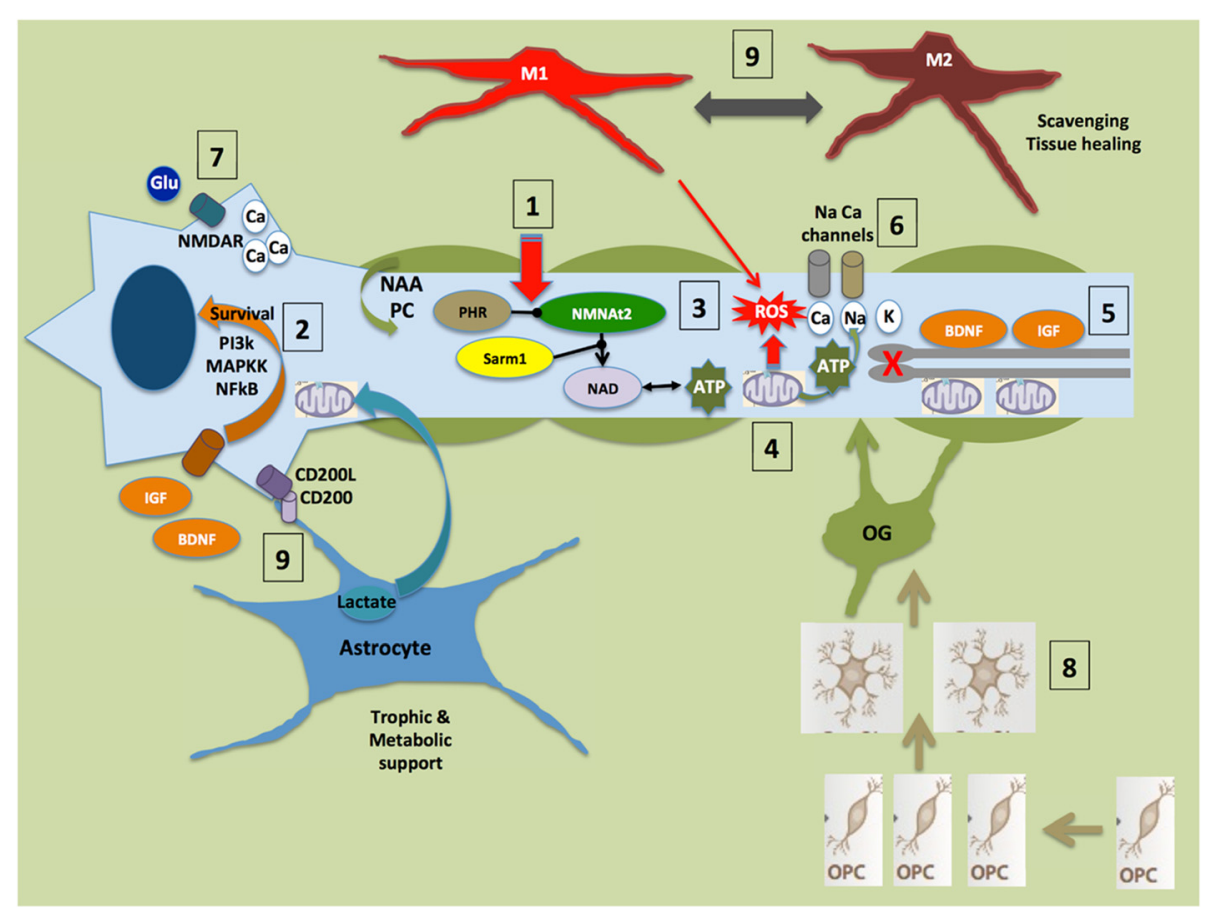

Fig. 1 Proposed targets for neuroprotective therapies. The pathways involved in neuroprotection include 1) Active axonal degeneration pathway activation (mediated by depletion of NAD levels by NMNAT2, PHR1 and Sarm1 activation); 2) trophic factor signaling (PI3K, MAPKK, NFKB); 3) oxidative stress (induced by inflammatory cells and mitochondria dysfunction); 4) energy depletion (due to mitochondria impairment and increased demand from (a channels); 5) axonal transport blockade (failing to deliver mitochondria, signaling and molecular complexes to soma, nodes of Ranvier or synpasis); 6) ionic imbalance (due to ion channel redistribution and changes in activity, leading to increase of intracellular calcium); 7) Excitotoxicity (mediated by excess of glutamate signaling through the NMDA receptors); 8) remyelination (from OPC repopulation of demyelinated areas to myelination of denuded axons by mature oligodendrocytes (OG), which provide metabolic support to the axon (NAA or PC); 9) protective effects of astrocytes (providing trophic factors such as IGF-1 or BDNF, metabolic substrates such as lactate, or pro-survival signals such as CD200-CD200L) and M2 microglia (with scavenger and tissue healing activity)

Table 1 Molecules and pathways targeted in neuroprotection

\begin{tabular}{|c|c|c|}
\hline Pathway & Molecule & Candidate drugs \\
\hline Trophic factors & BDNF, IGF-1, CNTF, etc. & rhBNDF, rhlGF-1, rhCNTF, etc., CERE-110 (AAV2-NGF) \\
\hline $\begin{array}{l}\text { Mitochondria dysfunction and } \\
\text { energy depletion }\end{array}$ & Cytochrome C, ATP & $\begin{array}{l}\text { Resveratrol, Rosiglitazone, Pioglitazone, Troglitazone, } \\
\text { Bezafibrate (PPARy activator) }\end{array}$ \\
\hline Ion channel & $\begin{array}{l}\text { Sodium, Potassium or Calcium channels, } \\
\text { Acid-Sensing lon Channels }\end{array}$ & Phenitoin, Lamotrigin, Amiloride \\
\hline Oxidative stress & iNOS, Nerf2 & $\begin{array}{l}\text { Dimethyl-Fumarate, Resveratrol, Vitamin E, Vitamin C, } \\
\text { Melatonin, Carnosine, Coenzyme-Q, Idebenone, Carotenoids }\end{array}$ \\
\hline Excitotoxicity & Glutamate & Memantine, Riluzole \\
\hline Demyelination & MOG, Lingo-1 & BIIB003, Clemastine benztropine, miconazole and clobetasol \\
\hline Axonal transport & Dynamin, kinesin, microtubules & Epothilone B \\
\hline $\begin{array}{l}\text { Inhibitory molecules axonal growth } \\
\text { and myelination }\end{array}$ & Lingo-1, Nogo-A & BIIB003, GSK1223249 \\
\hline Apoptosis & Bcl2, Bim, Bax, Cytochrome C, Caspase-3 & Caspase or calpain inhibitors, Mynocycline \\
\hline Microglia M2 mediated neuroprotection & $\begin{array}{l}\text { CD200, trophic factors (BDNF), } \\
\text { anti-inflammatory cytokines (IL-4, IL-10, TGFß) }\end{array}$ & $\begin{array}{l}\text { Interferon-beta, Glatiramer acetate, Fumarate, } \\
\text { Dimethyl-Fumarate, Mesenchymal stem cells }\end{array}$ \\
\hline Astrocyte mediated neuroprotection & Trophic factors, NAA, pyruvate, lactate & Pentamidine, Methylthioadenosine, Fingolimod \\
\hline
\end{tabular}


$[4,28,29]$. The CNS consumes $20 \%$ of the overall oxygen and energy of the body and neurons require the support of astrocytes for maintaining their metabolic activity [6]. Neurons use lactate as a substrate for the Krebs cycle, which is provided by astrocytes. Neurons are very sensitive to adenosine triphosphate (ATP) depletion and mitochondrial damage due to their highenergy consumption needed to maintain the intensive protein systems supporting their connections as well as the ion channels in charge of maintaining the electrical impulse. This is particularly true for axons, because they are long and tiny structures receiving support from the soma as well as from the axon-myelin unit [4]. Energy depletion, mitochondria function impairment and axonal transport deficits are common in CNS diseases and for this reason targeting energy supply to the CNS has been pursued as well. Studies began by providing additional sugar supplies or decreasing metabolic rate (e.g. hypothermia) but now other strategies such as administering metabolite precursors, or preserving mitochondria functioning have also been tested in trials [29-31]. There is now a strong interest in understanding the early molecular events in mitochondria damage during neuronal and axonal damage in order to prevent energetic failure $[4,7,32]$.

Ion channels are key for neuronal homeostasis and for maintaining the electrical impulse. Due to the highly specialized neuronal design, ion channel activity is highly prominent in the axonal initial segment as well as at the node of Ranvier [33]. This creates specific sites where ion fluxes are modulated and energy is required for axon functioning. Ion channel modulators have been explored as neuroprotective therapies in addition to their known beneficial effects in epilepsy or pain, including sodium channel modulators phenytoin, carbamacepin, lamotrigine, amiloride; and potassium channel modulators, aminopyridine or diazoxide (Table 2) [33]. Although benefits has been observed in animal models and small clinical trials, the challenge is determining how to maintain their beneficial effects in the long-term and understanding to which degree their effects are just maintaining the electrical impulse (symptomatic effects) versus promoting long-term neuronal or axonal survival (neuroprotection effects) [34].

Oxidative stress is another hot topic in neuroprotection [35]. The concept that free radicals degrade DNA and proteins suggest that anti-oxidative strategies would prevent cell death. There is ample evidence of the presence of increased oxidative stress in the damaged CNS in MS, neurodegenerative diseases, stroke and epilepsy [36-38]. However, oxidation in mitochondria and other organelles is a complex process required for energy production and other metabolic activities (e.g. signaling by nitric oxide (NO)) and for this reason is very difficult to modulate without inducing further damage. However, several approved therapies reduce oxidative stress induced by the insult such as the immunomodulatory drug dimethyl-fumarate (through activation of nuclear factor (erythroid-derived 2)-like 2 (Nerf2)) [39], and mesenchymal stem cells [40], which decrease reactive oxygen species induced by inflammation.

Excitotoxicity is postulated as the prime mechanism of damage in epilepsy and it has also been associated with neurodegenerative diseases as well with MS [41, 42]. By over-activating the excitatory glutamate receptors, neurons experience high levels of electrical and energetic activity, inducing ion imbalance and promoting neuronal death. Although there is clear evidence about the role of this process in animal models, its involvement in MS and other brain diseases has not been clarified in detail. Riluzole is an approved drug inhibiting N-methyl-Daspartate (NMDA) receptors, in addition to modulating sodium channels, but its efficacy in ALS is modest and trials in MS failed to show benefits [43]. Memantine is approved for Alzheimer's disease and was shown to modulate glutamate excitatory activation [44], but it was found to transiently worsen symptoms in patients with MS, reproducing pseudoexacerbations [45, 46].

Demyelination is the most prominent feature in MS. Promoting myelin recovery through remyelination is a natural strategy for treating demyelinating diseases. It is important to keep in mind that myelin is one of the most important elements for protecting axons, the myelin-axon unit [9]. Myelin is active not only in promoting saltatory conduction (which increases electrical conduction speed and reduces energy needs), but also in providing metabolic support to axons (e.g. N-AcetylAspartate (NAA), phosphatidylcholine (PC), etc.) [47]. For this reason, preventing demyelination and promoting remyelination is one of the most important neuroprotective strategies for MS [48]. There are several drugs being tested at present to promote remyelination by blocking leucine rich repeat and immunoglobin-like domain-containing protein 1 (Lingo-1) using anti-Lingo-1 monoclonal antibody, or repurposing drugs such as clemastine or guanabenz (Table 2). The main challenge will be to probe in humans whether such drugs are able to induce remyelination of the CNS and whether this biological activity translates to clinical benefits. Quantifying demyelination and remyelination in the living human CNS still remains a challenge at the clinical level due to technological limitations (e.g. lack of specificity of MRI sequences such as magnetic transfer ratio) [49].

Axonal transport is a key process in the homeostasis of neurons and their long connections. Axons need to transport to the synapsis most of the protein synthesis machinery as well as providing energy supply to the nodes of Ranvier and synapsis. In addition trophic factor 
Table 2 Neuroprotective drugs in clinical development for MS

\begin{tabular}{|c|c|c|c|c|c|c|}
\hline Drug & Company & Type of compound & MoA summary & $\begin{array}{l}\text { Route of } \\
\text { administration }\end{array}$ & Phase & CT.org \\
\hline KPT-350 & Karyopharm Therapeutic & $\begin{array}{l}\text { Small molecule - Selective Inhibitor } \\
\text { of Nuclear Export (SINE) }\end{array}$ & Antioxidant Neuroprotection & Oral & Preclinical & N/A \\
\hline NDC-1308 & ENDECE Neural & Small molecule Estradiol analog & Neuroprotection Remyelination & N/A & Preclinical & $\mathrm{N} / \mathrm{A}$ \\
\hline Methylthioadenosine & Digna Biotech & Metabolite & $\begin{array}{l}\text { Methyltransferases modulator Neuroprotection } \\
\text { Remyelination }\end{array}$ & Oral & Preclinical & N/A \\
\hline NRP2945 & CuroNZ & Peptide & Neuroprotection & N/A & Preclinical & N/A \\
\hline ER agonist & Karo Bio $A B$ & Smal chemicals & Estrogen Receptor beta agonist Neuroprotection & N/A & Preclinical & N/A \\
\hline$V \times 15 / 2503$ & Vaccinex & mAb - anti-semaphorin 4D & Anti-SEMA4D Neuroprotection Remyelination & Intravenous & Phase 1 & NCT01764737 \\
\hline RNS60 & Revalesio & Physically-Modified Saline & Immunomodulation Neuroprotection & Intravenous & Phase 2 & NCT01714089 \\
\hline GNbAC1 & GeNeuro & mAb First-in-Class & Immunomodulation Remyelination & Intravenous & Phase 2a & NCT01639300 \\
\hline TRO19622 Olesoxime & Trophos SA & Small chemical & Antioxidant & Oral & Phase 1 & NCT01808885 \\
\hline BIIB0033 - Anti-LINGO1 & Biogen Idec & $m A b$ & $\begin{array}{l}\text { LINGO-1 antagonist Remyelination } \\
\text { Neuroprotection }\end{array}$ & Intravenous & $\begin{array}{l}\text { Phase } 2 \\
\text { Phase } 2\end{array}$ & $\begin{array}{l}\text { NCT01864148 } \\
\text { NCT01721161 }\end{array}$ \\
\hline rHIgM22 & Acorda Therapeutics & mAb - Recombinant human IgM & Remyelination & Intravenous & Phase 1 & NCT01803867 \\
\hline MN-166 Ibudilast & MediciNova & Small molecule & Immunomodulation Neuroprotection & Oral & Phase 2 & NCT01982942 \\
\hline RGN-352 & RegeneRx & Peptide & Neuroprotection Remyelination & N/A & N/A & N/A \\
\hline EGCG - Epigallocatechin-gallate & Generic & Green tea extract (Polyphenon E) & Anti-oxidant & Oral & Phase 2 & $\begin{array}{l}\text { NCT00525668 } \\
\text { NCT01451723 }\end{array}$ \\
\hline Lamotrigine & GlaxoSmithKline & Small chemical & Sodium channel modulator Neuroprotection & Oral & Oral & NCT01879527 \\
\hline Phenitoin & Generic & Small chemical & Sodium channel modulator Neuroprotection & Oral & Phase 2 & NCT01451593 \\
\hline MRF-008 Guanabenz & $\begin{array}{l}\text { Myelin Repair } \\
\text { Foundation }\end{array}$ & Small chemical & $\begin{array}{l}\text { Alpha agonist of the alpha-2 adrenergic receptor } \\
\text { Remyelination }\end{array}$ & Oral & Phase 1 & NCT02423083 \\
\hline Clemastine & Generic & Small chemical & Remyelination & Oral & Phase 2 & NCT02040298 \\
\hline BAF312 & Novartis & Small chemical - Siponimod & S1P1 antagonist Neuroprotection & Oral & $\begin{array}{l}\text { Phase } 2 \\
\text { RRMS } \\
\text { Phase } 3 \\
\text { SPMS }\end{array}$ & NCT00879658 \\
\hline Amiloride & Generic & Small chemical & Sodium channel modulator & Oral & Phase 2 & NCT01910259 \\
\hline BN201 & Bionure & Small chemical & Neurotrophin agonist Neuroprotection & Intravenous & Phase 1 & N/A \\
\hline Erythropietin & Generic & Human recombinant protein & Trophic factor Neuroprotection & Intravenous & Phase 3 & NCT01962571 \\
\hline GSK1223249 - Ozanezumab & GlaxoSmithKline & $\mathrm{mAb}$ & Anti-Nogo-A Axonal regeneration & Intravenous & Phase 2 & NCT01435993 \\
\hline Diazoxide & Generic & Small chemical & $\begin{array}{l}\text { Potassium channel opener \& mitochondrial } \\
\text { channel modulator }\end{array}$ & Oral & Phase 2 & NCT01428726 \\
\hline Minocycline & Generic & Small chemical & Anti-apoptotic \& anti-oxidant & Oral & Phase 2 & NCT01073813 \\
\hline Riluzole & Generic & Small chemical & Sodium channel and NMDA modulator & Oral & Phase 2 & NCT00501943 \\
\hline
\end{tabular}


Table 2 Neuroprotective drugs in clinical development for MS (Continued)

\begin{tabular}{|c|c|c|c|c|c|c|}
\hline MD1003 - Biotin & Generic & Vitamin & $\begin{array}{l}\text { Carboxylases coenzyme (acetylCoA carboxylase) } \\
\text { Remyelination }\end{array}$ & Oral & Phase 3 & NCT02220933 \\
\hline BG12 - Dimethyl Fumarate & Biogen Idec & Metabolite & $\begin{array}{l}\text { Anti-oxidant hydroxycarboxylic acid receptor } 2 \\
\text { agonist }\end{array}$ & Oral & Approved & NCT00420212 \\
\hline FTY720 - Fingolimod & Novartis & Small chemical & S1P1 and S1P5 antagonist neuroprotection & Oral & Approved & NCT00355134 \\
\hline
\end{tabular}


transportation (e.g. brain-derived neurotrophic factor (BDNF)) from synapsis to the soma is also critical for neuronal survival [29]. This requires efficient transport through the axons based on intraxonal fluxes but mainly driven by microtubules transporting organelles to and from the soma using the dynein and kinesin systems respectively. Such molecular transporters are very sensitive to protein denaturation, lack of energy, inflammation and other types of damage [32, 50]. Also, it is known that mitochondria accumulate in the nodes of Ranvier (stationary sites) in order to provide extra ATP to sites rich in ion channels, and they are also transported to synapses to provide energy and are retrogradely transported for degradation. Inflammation, ischemia and other processes severely impair axonal transportation, including mitochondria delivery at nodes of Ranvier, contributing to energy and metabolite depletion and axonal damage $[32,51]$. Recently, it has been shown that tubuline targeting drugs may preserve microtubule function and protect axons from degradation in models of spinal cord injury [52]. In addition, neurofilaments are in charge of keeping the 3D axonal structure and after damage, neurofilaments become hyperphosphorylated, losing their function and inducing the collapse of axons [53]. Therefore, preserving axonal structure and function is an important strategy for promoting neuroprotection.

For more than a century, since Cajal seminal studies, it has been known that the brain inhibits axonal regrowth, which prevents CNS regeneration. The identification that myelin was the main inhibitor of axonal growth was followed by the identification of several proteins such as Lingo-1 and Nogo-A that prevent axonal growth signaling through the nerve growth factor (NGF) receptor p $75^{\mathrm{NGF}}$ receptor [54]. This mechanism is important for avoiding the formation of aberrant connections and preserving brain connectivity, at the cost of decreasing the regenerative capacity of the CNS. The discovery of the molecules responsible for such processes has been followed by the development of monoclonal antibodies (mAb) targeting these pathways in order to promote axonal regeneration. There are several clinical trials in MS testing such approaches including the use of $\mathrm{mAb}$ against Lingo-1 and Nogo-A (Table 2). The main concern is the pharmacological restrictions that $\mathrm{mAb}$ has in order to reach high levels in the CNS as well as the timing for this therapeutic intervention after injury.

Finally, it is well known that glia, including astrocytes and microglia, can display neuroprotective activities, although many of them are poorly understood $[12,55]$. For example, healthy neurons express CD200, which interacts with CD200L promoting survival signals [56]. Also, both microglia and astrocytes can release trophic factors and provide metabolic support to neuronal function [57]. The neuroprotective phenotype of microglia, also termed M2, is associated with suppressing inflammation and the creation of a supportive microenvironment supporting neuronal survival [12]. Furthermore, astrocytes, as the main supporters of neuronal function, display a wide array of positive functions for promoting neuronal survival. Moreover, stem cell therapy may show beneficial effects above and beyond just replacing cells by creating a supportive microenvironment and suppressing inflammation [12, 40]. Identification of the different mechanisms involved will provide new targets for developing neuroprotective strategies.

\section{Unmet needs for neuroprotection in MS and demyelinating diseases}

In MS, CNS damage is produced by a complex inflammatory process. Although in the past it was believed that in the relapsing-remitting phase CNS damage was due only to the presence of inflammatory infiltrates within the MS plaques, in the last decade it has been clearly shown that MS is a diffuse disease with inflammation, demyelination and axonal loss both in the grey and white matter [58, 59]. This diffuse inflammation, also termed trapped inflammation, is mainly drive by activated microglia, although cells of the adaptive immune system may be also present. Therefore, myelin and axons are acutely damaged by inflammatory infiltrates and chronically damaged by chronic microglia activation; both processes being present in relapsing and progressive MS to different extent and dynamics [36]. However, in progressive MS, relapses due to new inflammatory infiltrates tend to decrease or disappear because less tissue is available for damage. Also, in parallel to the inflammatory process, axonal degeneration takes place in CNS areas already damaged long time ago because of the lack of myelin support, presence of an aggressive microenvironment, retrograde axonal degeneration or transynaptic degeneration [60]. Moreover, after one decade of damage and recovery during the relapsing-remittig phase, oligodendrocytes fail to produce new myelin and ultimately die; with time, the capacity of oligodendrocyte precursors to replace lost oligodendrocytes also decreases, leading to large areas of demyelination [61]. In this scenario, it is clear that MS patients require therapies aimed at stopping the degenerative process and preventing new CNS damage, on top of the immunomodulatory strategy aimed at preventing inflammation [62].

Current immunomodulatory drugs are not completely effective and drugs with high efficacy may induce severe adverse-events. We must also take into account patient heterogeneity as well as the difficulty for predicting disease activity at the time of defining the immunomodulatory therapy. For this reason, at present is not possible to guarantee that treated patients are going to be free of disease activity and CNS damage induced by 
the autoimmune attack. And even in the best scenario, damaged tissue is still at risk of developing degenerative processes in the long-term. Considering that regenerative therapies are still far from being applied in clinical practice, protecting the brain against chronic inflammation (not significantly modulated by current immunotherapy), and preventing neurodegeneration in the long-term, is being pursued as the main strategy in the medium term for decreasing disability accumulation in patients with MS. This is true both for patients with relapsing MS as well as in the case of patients with progressive MS, in which inflammation is still present until the end and degenerative processes $[55,59]$.

In the case of other demyelinating diseases such as $\mathrm{NMO}$, the need for neuroprotection is also present but for different reasons [63]. In NMO there is no evidence of progressive course of the disease or presence of chronic trapped inflammation in the CNS. For this reason, all CNS damage and clinical disability observed in NMO is attributed to the damage induced during acute relapses, which are significantly more tissue destructive than in MS [64]. The necrotic characteristic of the NMO lesions has parallelism with stroke-induced damage, a prototypic model for neuroprotection in which is critical preventing severe CNS damage in order to reduce disability. The good news is that the inflammatory-induced damage in NMO may operate over longer periods of time than brain ischemia, providing a wider therapeutic window for intervention (from minutes to days). Recurrent Idiopatic Optic Neuritis, Relapsing Optic Neuritis or Transverse Myelitis are also other less common types of demyelinating diseases that would follow the NMO paradigm for neuroprotection, decreasing CNS damage due to relapses.

\section{Neuroprotective therapies under development for MS and other demyelinating diseases}

Neuroprotection is a well-accepted concept in the therapeutic strategy of neurologists, but in order to be useful at the clinical level, efficacy must be demonstrated in randomized clinical trials $[65,66]$. At present there is a growing list of new drugs and repurposing of drugs being tested from phase 1 to phase 3 trials (Table 2). One of the most active areas of research are remyelinating therapies, which can be categorized either as regenerative therapy (aimed to restore myelin) or neuroprotective therapy (aimed to protect axons and restore nerve conduction) [67]. A recent trial testing the mAb blocking Lingo-1 (BIIB033) has shown improvement in the latencies of the visual evoked potentials in patients with optic neuritis, suggesting an improvement of nerve conduction typically associated with remyelination (NCT01721161). Another mAb promoting remyelination is $\mathrm{rHIgM} 22$, which was discovered as part of the natural antibody repertoire in humans with remyelinating potential [68] and it is now being tested in clinical trials in MS patients (NCT01803867, NCT02398461). Regarding small chemicals, two approved drugs that are being explored for their effect on remyelination are clemastine and guanabenz after having shown a remyelinating effect in vitro and animal models [69].

Other strategies in clinical phases include the use of trophic factor compounds (eritropoietin, BN201), antioxidant compounds (the green tea extract epigallocatechin3-gallate, ginkgo biloba extracts, biotin, dimethyl-fumarate, olexosime), modulating estrogen receptors, metabolites (dimethyl-fumarate, methyltioadenosine), blocking semaphorins (VX15/2503), and ion channels modulators (carbamacepin, phenytoin, lamotrigin, amiloride, riluzole) (Table 2) [21, 70]. All these drugs still need to show their efficacy in phase 3 trials and then define how they would be integrated in the MS armamentarium, probably as a combination therapy with immunomodulators for relapsing MS or perhaps in combination with different agents for progressive MS.

In addition to drugs being tested as neuroprotectants, we must also consider stem cells as another approach to neuroprotection in MS [71]. At present, the most tested stem cells are mesenchymal stem cells, which have shown immunomodulatory and neuroprotective properties in vitro and in animal models [72]. Recent trials in patients with relapsing and progressive MS have shown some beneficial effects in terms of decreasing relapse rate or disability [73-75], but without clarifying whether these effects are due to its immunomodulatory or neuroprotective effects. A large multicentric randomized trial (MESEMS trial; NCT02403947) is ongoing to evaluate its efficacy in MS. In addition, phase 1 and 2 trials are ongoing or being planned to test the efficacy of other stem cells such as oligodendrocyte or olfactory ensheeting glial cells and probably neural cells in the near future.

\section{Challenges for developing neuroprotective therapies}

Lack of approved neuroprotective drugs is due to both poor understanding of the mechanisms of damage and the low recovery ability of the CNS (as discussed above) as well as the limitations of clinical trials for probing the efficacy of such drugs. First, probing the efficacy of a neuroprotective drug requires selecting the right indication, stage of the disease and group of patients in which such intervention can translate to a biological benefit as well as to a clinical benefit. For example, treating patients in the very late stages of the disease, when damage of the CNS is very severe and few neurons and axons can be rescued, may not translate to clinical benefits. This late diagnosis is one of the greatest limitations to date in other neurodegenerative diseases such as Alzheimer or Parkinson disease and probably in progressive MS. 
Second, pharmacological properties of the drug should be good enough to be sure to deliver the signal in the right site of the CNS and with enough intensity to obtain positive outcomes. This is one of the limitations of $\mathrm{mAb}$, stem cells and some other drugs. Also, dose selection and defining the therapeutic window based in efficacy-toxicity balance as well as in the timing of the intervention from the onset of damage is critical as well. Previous studies with recombinant trophic factors may have failed because poor pharmacokinetic properties as well as toxicity of the recombinant proteins prevented the use of efficacious doses [27]. Also, we must keep in mind that patients with MS are young and the disease evolves slowly over years, and for this reason MS patients are not likely to accept the risk of side-effects.

Third, biomarkers are envisioned as a key strategy for moving new drugs from preclinical stages to phase 1 and 2 trials, helping to select the best therapeutic regimen and dose, identify the best patient subgroups to be tested (avoiding non-responders) and match surrogate with clinical endpoints in order to optimize trials results [76]. Also, systems medicine is going to help in the integration of biological and clinical knowledge in a more comprehensive understanding of the disease and patients' heterogeneity, which will pay off by improving our accuracy for transition from preclinical to clinical stages of drug development $[77,78]$.

Finally, in order to probe efficacy of neuroprotective drugs in clinical trials, we need sensitive surrogate and clinical endpoints for the mechanism of action and the level of damage. Again, this is critical because many drugs may have failed not due to a lack of efficacy, but because the incapacity to measure such effects with the proposed end-points. The most common proposed surrogate endpoints is imaging. In the case of MRI, the most validated marker for MS is presence of new lesions (either contrast enhancing lesions or new lesions in T2), but this marker is useful for immunomodulatory drugs, not for neuroprotective drugs. Alternatively, brain atrophy is the best correlate of disability in MS, but brain atrophy is difficult to measure requiring advanced imaging methods, prone to high variability between scanners and techniques and with low sensitivity to changes [79]. For this reason, other approaches such as optical coherence tomography offer the opportunity to quantify with high accuracy retina atrophy [80] and is now being added to phase 2 and 3 clinical trials [81]. Regarding clinical endpoints, clinical scales such as the Expanded Disability Status Scale (EDSS) are complex, with high variability and subjectivity and with low sensitivity to meaningful clinical changes associated with neuroprotection. For this reason, several new clinical outcomes such as low contrast visual acuity [82] or activity levels measured with accelerometers [83] are promising avenues for evaluating neuroprotective drugs.

\section{Conclusions}

Relapsing MS and other demyelinating diseases have benefited significantly from the advancements of new immunomodulatory therapies, but the challenge of protecting CNS from damage remains one of the top priorities for all demyelinating diseases as well as neurodegenerative diseases or acute brain damage by trauma or stroke. Novel discoveries in neurobiology provide new therapeutic targets, and new imaging modalities provide the opportunity to evaluate the efficacy of these new neuroprotective drugs. Because several of the mechanisms being targeted by neuroprotective therapies are common between diseases, some of the biomarkers and therapeutics strategies may be useful for different type of diseases, although medicine use to tell us that any single therapeutic approach fitting all CNS diseases is highly unlikely.

We will hopefully soon have effective neuroprotective therapies ready to use in patients, which combined with immunomodulatory drugs, will help to prevent CNS damage, decrease neurological disability and improve MS patients' quality of life. Although several therapeutic regimens can be proposed, neuroprotective therapy is envisioned as combination therapy with other disease modifying drugs targeting the pathogenic cascade, such as immunomodulatory therapy in MS. Neuroprotective therapies should be started early in the course of the disease, because axonal damage start to accumulate from the beginning of the disease. And such therapies may extent for the whole life and for almost all types of disease, from clinically isolated syndromes to progressive MS. In addition to the use of neuroprotective therapies for preventing chronic damage in MS, these therapies would be used for acute neuroprotection at the time patients suffer an acute relapse of MS, NMO or Optic Neuritis. However, we need to learn which neuroprotective therapy is more required for each subgroup of patients to be more effective. To this aim, it is required development of biomarkers of CNS damage processes, to be used to select the right drugs at each stage of the disease process. This may also help to identify MS subtypes with different involvement of CNS damage mechanisms operating at a given time. Such subtypes may overlap with MS pathological subtypes or with genetic risks, which is unknown at present. Tailoring combination therapy with immunotherapies using biomarkers would be one of the next challenges for MS therapeutics in the medium term that hopefully is going to improve the quality of life of people with MS.

\section{Abbreviations}

ALS: amiotrophic lateral sclerosis; ATP: adenosin tri-phosphate; BDNF: brain derived nerve factor; CNS: central nervous system; EDSS: expanded disability status scale; GSK3: glycogen synthase kinase 3; IKK: inhibitor of kappa B kinase; JNK: c-Jun N-terminal kinases; Lingo-1: leucine rich repeat and 
immunoglobin-like domain-containing protein 1; mAb: monoclonal antibody; MAPKK: mitogen-activated protein kinase kinase; Nerf2: nuclear factor (erythroid-derived 2)-like 2; NMDA: N-methyl-D-aspartate; MS: multiple sclerosis; NMO: neuromyelitis optica; NO: nitric oxide; MRI: magnetic resonance imaging; NAA: N-Acetyl- Aspartate; NAD: nicotinamide adenine dinucleotide; NFKB: nuclear factor-KB; Nogo-A: neurite outgrowth inhibitor $A_{\text {; }}$ NMNAT2: nicotinamide nucleotide adenylyltransferase 2; PC: phosphatidilcholine; PI3K: phosphoinositide 3-kinase; PHR1: phosphate starvation response 1; Sarm1: Sterile Alpha And TIR Motif Containing 1.

\section{Competing interests}

PV has received consultancy fees from Roche, Novartis and Digna Biotech. He is founder and advisor of Bionure Inc. and holds patent rights for the use of Methylthioadenosine and BN201 for the treatment of MS and other neurological diseases.

\section{Acknowledgement}

I would like to thanks Erika Lampert for the English review of the manuscript. This work was supported by the Instituto de Salud Carlos III, Spain (FIS: PI12/01823) to PV.

\section{Received: 26 August 2015 Accepted: 17 February 2016 Published online: 01 April 2016}

\section{References}

1. Hagmann P, Sporns O, Madan N, Cammoun L, Pienaar R, Wedeen VJ, et al. White matter maturation reshapes structural connectivity in the late developing human brain. Proc Natl Acad Sci U S A. 2010;107(44):19067-72.

2. Nave KA, Werner HB. Myelination of the nervous system: mechanisms and functions. Annu Rev Cell Dev Biol. 2014;30:503-33.

3. Franklin RJ, ffrench-Constant C, Edgar JM, Smith KJ. Neuroprotection and repair in multiple sclerosis. Nat Rev Neurol. 2012;8(11):624-34.

4. Court FA, Coleman MP. Mitochondria as a central sensor for axonal degenerative stimuli. Trends Neurosci. 2012;35(6):364-72.

5. Lassmann $\mathrm{H}$. Axonal and neuronal pathology in multiple sclerosis: what have we learnt from animal models. Exp Neurol. 2010;225(1):2-8.

6. Harris JJ, Attwell D. The energetics of CNS white matter. J Neurosci. 2012;32(1):356-71.

7. Conforti L, Gilley J, Coleman MP. Wallerian degeneration: an emerging axon death pathway linking injury and disease. Nat Rev Neurosci. 2014;15(6):394-409.

8. Adalbert R, Coleman MP. Review: Axon pathology in age-related neurodegenerative disorders. Neuropathol Appl Neurobiol. 2013;39(2):90-108.

9. Nave KA. Myelination and support of axonal integrity by glia. Nature. 2010;468(7321):244-52.

10. Jurgens T, Jafari M, Kreutzfeldt M, Bahn E, Bruck W, Kerschensteiner M, et al. Reconstruction of single cortical projection neurons reveals primary spine loss in multiple sclerosis. Brain. 2015:139(Pt 1):39-46

11. Lipton SA. Pathologically activated therapeutics for neuroprotection. Nat Rev Neurosci. 2007:8(10):803-8.

12. Giunti D, Parodi B, Cordano C, Uccelli A, Kerlero de Rosbo N. Can we switch microglia's phenotype to foster neuroprotection? Focus on multiple sclerosis. Immunology. 2014;141(3):328-39.

13. Colombo E, Di Dario M, Capitolo E, Chaabane L, Newcombe J, Martino G, et al. Fingolimod may support neuroprotection via blockade of astrocyte nitric oxide. Ann Neurol. 2014;76(3):325-37.

14. Reick C, Ellrichmann G, Thone J, Scannevin RH, Saft C, Linker RA, et al. Neuroprotective dimethyl fumarate synergizes with immunomodulatory interferon beta to provide enhanced axon protection in autoimmune neuroinflammation. Exp Neurol. 2014;257:50-6.

15. Ruffini F, Rossi S, Bergamaschi A, Brambilla E, Finardi A, Motta C, et al. Laquinimod prevents inflammation-induced synaptic alterations occurring in experimental autoimmune encephalomyelitis. Mult Scler. 2013;19(8):1084-94.

16. Aharoni R. Immunomodulation neuroprotection and remyelination - the fundamental therapeutic effects of glatiramer acetate: a critical review. J Autoimmun. 2014:54:81-92

17. Loeb JA. Neuroprotection and repair by neurotrophic and gliotrophic factors in multiple sclerosis. Neurology. 2007;68(22 Suppl 3):S38-42. discussion S3-54.

18. Villoslada P, Genain CP. Role of nerve growth factor and other trophic factors in brain inflammation. Prog Brain Res. 2004;146:403-14.
19. Segal RA. Selectivity in neurotrophin signaling: theme and variations. Annu Rev Neurosci. 2003:26:299-330.

20. Villoslada P, Hauser SL, Bartke I, Unger J, Heald N, Rosenberg D, et al. Human nerve growth factor protects common marmosets against autoimmune encephalomyelitis by switching the balance of $T$ helper cell type 1 and 2 cytokines within the central nervous system. J Exp Med. 2000;191(10):1799-806

21. Colafrancesco V, Villoslada P. Targeting NGF pathway for developing neuroprotective therapies for multiple sclerosis and other neurological diseases. Arch Ital Biol. 2011;149(2):183-92.

22. The BDNF Study Group (Phase III). A controlled trial of recombinant methionyl human BDNF in ALS: Neurology. 1999;52(7):1427-33.

23. ALS CNTF Treatment Study Group. A double-blind placebo-controlled clinical trial of subcutaneous recombinant human ciliary neurotrophic factor (rHCNTF) in amyotrophic lateral sclerosis. Neurology. 1996:46(5):1244-9.

24. Sorenson EJ, Windbank AJ, Mandrekar JN, Bamlet WR, Appel SH, Armon C, et al. Subcutaneous IGF-1 is not beneficial in 2-year ALS trial. Neurology. 2008;71(22):1770-5.

25. Tuszynski MH, Thal L, Pay M, Salmon DP, U HS, Bakay R, et al. A phase 1 clinical trial of nerve growth factor gene therapy for Alzheimer disease. Nat Med. 2005;11(5):551-5.

26. Cudkowicz ME, Katz J, Moore DH, O'Neill G, Glass JD, Mitsumoto H, et al. Toward more efficient clinical trials for amyotrophic lateral sclerosis. Amyotroph Lateral Scler. 2010;11(3):259-65.

27. Apfel SC. Nerve growth factor for the treatment of diabetic neuropathy: what went wrong, what went right, and what does the future hold? Int Rev Neurobiol. 2002;50:393-413.

28. Beal MF. Mitochondria take center stage in aging and neurodegeneration. Ann Neurol. 2005;58(4):495-505.

29. Schapira AH, Olanow CW, Greenamyre JT, Bezard E. Slowing of neurodegeneration in Parkinson's disease and Huntington's disease: future therapeutic perspectives. Lancet. 2014;384(9942):545-55.

30. Tiwari SK, Chaturvedi RK. Peptide therapeutics in neurodegenerative disorders. Curr Med Chem. 2014:21(23):2610-31.

31. Ries $V$, Oertel $W H$, Hoglinger GU. Mitochondrial dysfunction as a therapeutic target in progressive supranuclear palsy. J Mol Neurosci. 2011:45(3):684-9.

32. Errea O, B M, Gonzalez-Franquesa A, Garcia-Roves P, Villoslada P. The disruption of mitochondrial axonal transport is an early event in neuroinflammation. J Neuroinflammation. 2015;12:152-167.

33. Waxman SG. Axonal conduction and injury in multiple sclerosis: the role of sodium channels. Nat Rev Neurosci. 2006;7(12):932-41.

34. Waxman SG. Mechanisms of disease: sodium channels and neuroprotection in multiple sclerosis-current status. Nat Clin Pract Neurol. 2008:4(3):159-69.

35. Sureda FX, Junyent F, Verdaguer E, Auladell C, Pelegri C, Vilaplana J, et al. Antiapoptotic drugs: a therapautic strategy for the prevention of neurodegenerative diseases. Curr Pharm Des. 2011;17(3):230-45.

36. Lassmann H. Multiple sclerosis: Lessons from molecular neuropathology. Exp Neurol. 2014;262PA:2-7.

37. Haider L, Fischer MT, Frischer JM, Bauer J, Hoftberger R, Botond G, et al, Oxidative damage in multiple sclerosis lesions. Brain. 2011;134(Pt 7):1914-24.

38. Niedzielska E, Smaga I, Gawlik M, Moniczewski A, Stankowicz P, Pera J, et al. Oxidative Stress in Neurodegenerative Diseases. Mol Neurobiol. 2015. in press.

39. Linker RA, Lee DH, Ryan S, van Dam AM, Conrad R, Bista P, et al. Fumaric acid esters exert neuroprotective effects in neuroinflammation via activation of the Nrf2 antioxidant pathway. Brain. 2011;134(Pt 3):678-92.

40. Lanza C, Morando S, Voci A, Canesi L, Principato MC, Serpero LD, et al. Neuroprotective mesenchymal stem cells are endowed with a potent antioxidant effect in vivo. J Neurochem. 2009;110(5):1674-84.

41. Fern RF, Matute C, Stys PK. White matter injury: Ischemic and nonischemic. Glia. 2014;62(11):1780-9.

42. Kostic M, Zivkovic N, Stojanovic I. Multiple sclerosis and glutamate excitotoxicity. Rev Neurosci. 2013;24(1):71-88.

43. Mitsumoto H. Riluzole-what is its impact in our treatment and understanding of amyotrophic lateral sclerosis? Ann Pharmacother. 1997;31(6):779-81.

44. Lipton SA. Paradigm shift in neuroprotection by NMDA receptor blockade: Memantine and beyond. Nat Rev Drug Discov. 2006;1-11.

45. Villoslada P, Arrondo G, Sepulcre J, Alegre M, Artieda J. Memantine induces reversible neurologic impairment in patients with MS. Neurology. 2008;72(19):1630-3.

46. Green AJ. Understanding pseudo: the symptoms are real, the cause is unclear. Neurology. 2009;72(19):1626-7. 
47. Nave KA, Trapp BD. Axon-glial signaling and the glial support of axon function. Annu Rev Neurosci. 2008;31:535-61.

48. DeLuca GC, Williams K, Evangelou N, Ebers GC, Esiri MM. The contribution of demyelination to axonal loss in multiple sclerosis. Brain. 2006;129(Pt 6):1507-16.

49. van den Elskamp IJ, Knol DL, Vrenken H, Karas G, Meijerman A, Filippi M, et al. Lesional magnetization transfer ratio: a feasible outcome for remyelinating treatment trials in multiple sclerosis. Mult Scler. 2010;16(6):660-9.

50. De Vos KJ, Grierson AJ, Ackerley S, Miller CC. Role of axonal transport in neurodegenerative diseases. Annu Rev Neurosci. 2008;31:151-73.

51. Sorbara CD, Wagner NE, Ladwig A, Nikic I, Merkler D, Kleele T, et al. Pervasive axonal transport deficits in multiple sclerosis models. Neuron. 2014;84(6):1183-90.

52. Ruschel J, Hellal F, Flynn KC, Dupraz S, Elliott DA, Tedeschi A, et al. Axonal regeneration. Systemic administration of epothilone B promotes axon regeneration after spinal cord injury. Science. 2015;348(6232):347-52.

53. Kim S, Chang R, Teunissen C, Gebremichael Y, Petzold A. Neurofilament stoichiometry simulations during neurodegeneration suggest a remarkable self-sufficient and stable in vivo protein structure. J Neurol Sci. 2011;307(1-2):132-8.

54. Llorens F, Gil V, del Rio JA. Emerging functions of myelin-associated proteins during development, neuronal plasticity, and neurodegeneration. FASEB J. 2011;25(2):463-75.

55. Rottlaender A, Kuerten S. Stepchild or Prodigy? Neuroprotection in Multiple Sclerosis (MS) Research. Int J Mol Sci. 2015;16(7):14850-65.

56. Hernangomez M, Mestre L, Correa FG, Loria F, Mecha M, Inigo PM, et al. CD200-CD200R1 interaction contributes to neuroprotective effects of anandamide on experimentally induced inflammation. Glia. 2012;60(9):1437-50.

57. Vecino E, Rodriguez FD, Ruzafa N, Pereiro X, Sharma SC. Glia-neuron interactions in the mammalian retina. Prog Retin Eye Res. 2015;51:1-40.

58. Ransohoff RM, Hafler DA, Lucchinetti CF. Multiple sclerosis-a quiet revolution. Nat Rev Neurol. 2015;11(3):134-42.

59. Mahad DH, Trapp BD, Lassmann H. Pathological mechanisms in progressive multiple sclerosis. Lancet Neurol. 2015;14(2):183-93.

60. Lassmann H. Pathology and disease mechanisms in different stages of multiple sclerosis. J Neurol Sci. 2013;333(1-2):1-4.

61. Piaton G, Gould RM, Lubetzki C. Axon-oligodendrocyte interactions during developmental myelination, demyelination and repair. J Neurochem. 2010;114(5):1243-60.

62. Mullard A. Success of immunomodulators in MS shifts discovery focus to neuroprotection. Nat Rev Drug Discov. 2011;10(12):885-7.

63. Weinshenker BG, Barron G, Behne JM, Bennett JL, Chin PS, Cree BA, et al. Challenges and opportunities in designing clinical trials for neuromyelitis optica. Neurology. 2015;84(17):1805-15.

64. Pittock SJ, Lucchinetti CF. Neuromyelitis optica and the evolving spectrum of autoimmune aquaporin-4 channelopathies: a decade later. Ann N Y Acad Sci. 2015.in press.

65. Maghzi AH, Minagar A, Waubant E. Neuroprotection in multiple sclerosis: a therapeutic approach. CNS Drugs. 2013:27(10):799-815.

66. Aktas O, Kieseier B, Hartung HP. Neuroprotection, regeneration and immunomodulation: broadening the therapeutic repertoire in multiple sclerosis. Trends Neurosci. 2010;33(3):140-52.

67. Luessi F, Kuhlmann T, Zipp F. Remyelinating strategies in multiple sclerosis. Expert Rev Neurother. 2014;14(11):1315-34.

68. Mitsunaga Y, Ciric B, Van Keulen V, Warrington AE, Paz Soldan M, Bieber AJ, et al. Direct evidence that a human antibody derived from patient serum can promote myelin repair in a mouse model of chronic-progressive demyelinating disease. FASEB J. 2002;16(10):1325-7.

69. Mei F, Fancy SP, Shen YA, Niu J, Zhao C, Presley B, et al. Micropillar arrays as a high-throughput screening platform for therapeutics in multiple sclerosis. Nat Med. 2014;20(8):954-60.

70. Raftopoulos RE, Kapoor R. Neuroprotection for acute optic neuritis-Can it work? Mult Scler Relat Disord. 2013;2(4):307-11.

71. Payne N, Siatskas C, Barnard A, Bernard CC. The prospect of stem cells as multi-faceted purveyors of immune modulation, repair and regeneration in multiple sclerosis. Curr Stem Cell Res Ther. 2011:6(1):50-62.

72. Uccelli A, Moretta L, Pistoia V. Mesenchymal stem cells in health and disease. Nat Rev Immunol. 2008:8(9):726-36.

73. Llufriu S, Sepulveda M, Blanco Y, Marin P, Moreno B, Berenguer J, et al Randomized placebo-controlled phase II trial of autologous mesenchymal stem cells in multiple sclerosis. PLoS One. 2014;9(12), e113936.
74. Connick P, Kolappan M, Crawley C, Webber DJ, Patani R, Michell AW, et al. Autologous mesenchymal stem cells for the treatment of secondary progressive multiple sclerosis: an open-label phase 2a proof-of-concept study. Lancet Neurol. 2012;11(2):150-6.

75. Karussis D, Karageorgiou C, Vaknin-Dembinsky A, Gowda-Kurkalli B, Gomori JM, Kassis I, et al. Safety and immunological effects of mesenchymal stem cell transplantation in patients with multiple sclerosis and amyotrophic lateral sclerosis. Arch Neurol. 2010;67(10):1187-94.

76. Villoslada P. Biomarkers for multiple sclerosis. Drug News Perspect. 2010;23(9):585-95.

77. Villoslada P, Steinman L, Baranzini SE. Systems biology and its application to the understanding of neurological diseases. Ann Neurol. 2009;65(2):124-39.

78. Villoslada P, Baranzini S. Data integration and systems biology approaches for biomarker discovery: Challenges and opportunities for multiple sclerosis. J Neuroimmunol. 2012;248(1-2):58-65.

79. Barkhof F, Calabresi PA, Miller DH, Reingold SC. Imaging outcomes for neuroprotection and repair in multiple sclerosis trials. Nat Rev Neurol. 2009;5(5):256-66.

80. Martinez-Lapiscina E, Sanchez-Dalmau B, Fraga-Pumar E, Ortiz-Perez S, Tercero-Uribe A, Torres-Torres $\mathrm{R}$, et al. The visual pathway as a model to understand brain damage in multiple sclerosis. Mult Scler. 2014:20(13):1678-85.

81. Zarbin MA, Jampol LM, Jager RD, Reder AT, Francis G, Collins W, et al. Ophthalmic evaluations in clinical studies of fingolimod (FTY720) in multiple sclerosis. Ophthalmology. 2013;120(7):1432-9.

82. Balcer LJ. Clinical trials to clinical use: using vision as a model for multiple sclerosis and beyond. J Neuroophthalmol. 2014;34(Suppl):S18-23.

83. Sola-Valls N, Blanco Y, Sepulveda M, Llufriu S, Martinez-Lapiscina EH, La Puma $D$, et al. Walking function in clinical monitoring of multiple sclerosis by telemedicine. J Neurol. 2015;262(7):1706-13.

\section{Submit your next manuscript to BioMed Central and we will help you at every step:}

- We accept pre-submission inquiries

- Our selector tool helps you to find the most relevant journal

- We provide round the clock customer support

- Convenient online submission

- Thorough peer review

- Inclusion in PubMed and all major indexing services

- Maximum visibility for your research

Submit your manuscript at www.biomedcentral.com/submit
) Biomed Central 